\title{
Relation between immune variables and burnout in a sample of physicians
}

\author{
Annalisa Bargellini, Alberto Barbieri, Sergio Rovesti, Roberto Vivoli, Roberto Roncaglia, \\ Paola Borella
}

\begin{abstract}
Objectives-To evaluate in a group of physicians the relation between burnout, demographic or job characteristics, anxiety, and immune variables.

Methods-Seventy one physicians of all grades were recruited among different departments to a cross sectional survey. The Maslach burnout inventory, scales of emotional exhaustion, depersonalisation, and personal accomplishment, the trait scale of anxiety inventory (STAI-Y2), and a questionnaire on personal and professional characteristics were administered. The immune profile included quantitative (number (\%) of lymphocytes and subsets) and functional (natural killer cytotoxicity) measures.
\end{abstract}

Results-With a model of stepwise multiple regression analysis, emotional exhaustion was significantly affected by both personal (marital, sex) and job characteristics (qualification, working activity), whereas only patient contact explained a portion of variation in depersonalisation. Furthermore, trait anxiety was found to predict the Maslach burnout inventory scores. After correction for potential confounders, physicians who scored high levels of personal accomplishment showed significantly higher numbers of total lymphocytes, T cells (CD3), T helper cells (CD4), and T suppressor cells (CD8) than those who scored low levels. No other correlation was found between burnout and immune variables.

Dipartimento di Scienze Igienistiche, Microbiologiche e Biostatistiche, Via Campi 287, I-41100 Modena, Italy

A Bargellini

S Rovesti

R Vivoli

P Borella

Department of Emergency Care A Barbieri

Department of Immune-haematology and Transfusion, Azienda Policlinico, Modena, Italy

R Roncaglia

Correspondence to: Professor Paola Borella borella@unimo.it

Accepted 25 February 2000
In occupational health research, topics on work stress have been identified as a priority. ${ }^{1}$ Indeed, the prevalence of exposure to psycho- logical overload and the corresponding health effects have increased during the past decades and will probably increase even further in the near future. ${ }^{2}$ Healthcare personnel are particularly at risk of developing work related psychological disorders, impacting negatively on patient care. Furthermore, it has been suggested that recent reforms in the National Health System threaten to increase emotional distress and decrease satisfaction that doctors derive from their work. ${ }^{3}$

The outcome of continued exposure to overwork, resulting in a progressive inability to carry out the responsibilities of the job has been termed burnout. For the assessment of the syndrome, still not recognised as illness, one of the more widely known models is that proposed by Maslach and Jackson. ${ }^{4}$ The Maslach burnout inventory is a short, easily administered measure with good reliability and may be useful for appraising levels of work related strain in different work environments.

The inventory has been largely used on healthcare providers and the link between burnout and several salient, work related, personal criteria has been described..$^{5-15}$ Several important issues on its underlying causes and biological effects remain to be considered. As far as we know, no publications are available on the involvement of cellular immunity in the burnout syndrome, except for a recent study on natural killer cells in a group of male office workers. ${ }^{16}$ Chronic psychological stress has been suggested to alter several immunological processes and exhaustive reviews have recently been published about this. ${ }^{17-21}$ Moreover, it has been proved that personality factors and social characteristics, such as anxiety, emotional stability, depression, loneliness, coping reaction style, and social support, render people more or less susceptible to stress induced immune changes. ${ }^{22-27}$ Recent studies suggest that burnout also may reflect enduring qualities of the person, and not only a stress reaction induced by the situation. Indeed, different personality factors accounted for a significant portion of the variation in burnout scores, ${ }^{28}$ and anxiety as well as other personality traits were significant factors in outlining a profile of highly burned out health workers. ${ }^{29}$

The aims of this study were as follows: $(a)$ to measure burnout in a group of physicians; $(b)$ to evaluate demographic and professional variables able to predict the level of distress; (c) to assess the contribution of trait anxiety to the experience of burnout; (d) to investigate the association of the individual degree of burnout with several immune variables. 


\section{Materials and methods}

The sample examined consisted of 71 physicians from three different hospitals in Modena (Italy); 51 of them were working in anaesthesiology and intensive care, and 20 were organising health policy and management, not involved directly with patients. They represent the $78.9 \%$ of the initial sample of 90 approached. Eight were excluded because they were $>50$ years of age or had been absent from service in the past 6 months, the others did not respond. Participants were invited to a meeting about the purpose of the study, and to explain in detail the arrangements for confidentiality and management of data, to answer questions, and gain cooperation.

The protocol included the Maslach burnout inventory and the trait scale of anxiety inventory (STAI-Y2), Italian versions, to assess the levels of burnout and trait anxiety, respectively. ${ }^{30} 31$ The Maslach burnout inventory consists of three scales: emotional exhaustion referring to the emotional stress and exhaustion at work, depersonalisation evaluating the development of emotional isolation at work, personal accomplishment evaluating the degree of satisfaction with work. The Italian version of the Maslach burnout inventory reports the expected levels for the Italian health workers, obtained on a representative group of 748 people, ${ }^{30}$ whereas STAI-Y2 describes the expected values for the Italian population divided according to sex. ${ }^{31}$ These normative data were used to indirectly compare results from our sample. A third questionnaire collected detailed information on demographic and professional characteristics, and on lifestyle (smoking habits, alcohol, drug, and coffee intake, sleeping habits, physical activity). Body mass index was calculated as weight $(\mathrm{kg})$ / height $\left(\mathrm{m}^{2}\right)$ The subjects completed questionnaires at home in one sitting and returned them in an anonymous form.

Blood samples $(15 \mathrm{ml})$ were taken between 0700 and 0800, after a free weekend and a peaceful night, and before beginning their working activity. The subjects were asked to sit quietly for a 15 minute period, and to avoid smoking or drinking before taking blood. Red and white cells were counted with a Coulter device. Lymphocyte subpopulations were measured with Coulter monoclonal antibodies and an indirect immunofluorescence technique standardised for Italian blood donors. ${ }^{32}$ Measured variables were the pan $\mathrm{T}$ cell population (CD3), $\mathrm{T}$ helper cells (CD4), $\mathrm{T}$ suppressor cells (CD8), CD4/CD8, B cells (CD19), and natural killer cells (CD16 ${ }^{+}$ $\mathrm{CD}^{-}$). The natural killer (NK) cell activity was measured with a 4 hour non-radioactive $\mathrm{Cr}$ release assay from labelled K562 tumour cells, adopting a new method standardised within our laboratory. ${ }^{33} 34$

At the end of the study, participants received a letter with their individual results and a brief summary report. A further meeting was organised to communicate general results, and to give further explanation of their results at a personal level.

Statistical analyses were performed with
SPSS version 8.0. Normality of data distribution was tested by Kolmogorov-Smirnov test; when the distribution was not normal, a log transformation of data was applied. Unpaired Student's $t$ test and one way analysis of variance (ANOVA) were applied to test the effects of personal and professional variables on the burnout scores. Based on results obtained by descriptive analyses, a stepwise multiple regression analysis was applied. In a first model, scores of for each Maslach burnout inventory scale were separately introduced as dependent variables, whereas independent ones were the demographic and professional characteristics associated with the Maslach burnout inventory scales in the univariate analysis. The introduction of all the variables considered did not change the results. The variables considered were: sex (male, female), age (years), marital status (single, married), children at home (n), working activity (patient contact no, yes), job position (vice head, assistant, IV, III, and II years postgraduate), and duration of service (years). In a second model, the anxiety score was added to the predictors. Finally, anxiety was subjected to regression analysis to evaluate demographic and job characteristics able to affect this enduring personal quality. Pearson's correlations were performed to evaluate the relation between the Maslach burnout inventory scores, anxiety, and immune variables. Stepwise multiple regression analyses were also applied to test for associations between immune variables and the burnout scores. Each immune test was separately introduced as a dependent variable, whereas independent ones were the Maslach burnout inventory and anxiety scores; potential confounders were sex, age, smoking habits (cigarettes/day), duration of service, and job position, which were associated with either the Maslach burnout inventory scales or distribu-

Table 1 Demographic and professional characteristics of the sample studied

\begin{tabular}{|c|c|c|c|}
\hline & $n$ & $\%$ & \\
\hline \multicolumn{4}{|l|}{ Sex: } \\
\hline Male & 39 & 54.9 & \\
\hline Female & 32 & 45.1 & \\
\hline \multicolumn{4}{|l|}{ Marital status: } \\
\hline Single & 37 & 52.1 & \\
\hline Married & 34 & 47.9 & \\
\hline \multicolumn{4}{|l|}{ Children at home: } \\
\hline 0 & 43 & 60.6 & \\
\hline 1 & 10 & 14.1 & \\
\hline 2 & 17 & 23.9 & \\
\hline 3 & 1 & 1.4 & \\
\hline \multicolumn{4}{|l|}{ Type of work: } \\
\hline Patient contact & 51 & 71.8 & \\
\hline No patient contact & 20 & 28.2 & \\
\hline \multicolumn{4}{|l|}{ Job position: } \\
\hline Vice head & 17 & 23.9 & \\
\hline Assistant & 19 & 26.8 & \\
\hline 4 y postgraduate & 9 & 12.7 & \\
\hline 3 y postgraduate & 16 & 22.5 & \\
\hline 2 y postgraduate & 10 & 14.1 & \\
\hline \multicolumn{4}{|l|}{ Years with the service: } \\
\hline$>5$ & 39 & 54.9 & \\
\hline $5-9$ & 13 & 18.3 & \\
\hline \multirow[t]{2}{*}{$>9$} & 19 & 26.8 & \\
\hline & Mean & $S D$ & Range \\
\hline Age $(y)$ & 35.4 & 5.8 & $24-50$ \\
\hline \multicolumn{4}{|l|}{ BMI $\left(\mathrm{kg} / \mathrm{m}^{2}\right)$ : } \\
\hline Male & 24.30 & 2.67 & $19.71-24.41$ \\
\hline Female & 21.67 & 2.90 & $16.85-29.39$ \\
\hline Duration of service (y) & 6.65 & 6.07 & $1-26$ \\
\hline
\end{tabular}


Table 2 Maslach burnout inventory (MBI): scores of emotional exhaustion (EE), depersonalisation $(D P)$, and personal accomplishment $(P A)$ scales

\begin{tabular}{|c|c|c|c|c|c|}
\hline & \multicolumn{2}{|l|}{ Scores } & \multicolumn{3}{|c|}{ Subjects (\%) with burnout level ${ }^{\star}$} \\
\hline & Mean (SD) & Range & Low & Medium & High \\
\hline \multicolumn{6}{|l|}{ MBI-EE: } \\
\hline Male (39) & $14.33(7.83)$ & $0-34$ & 51.3 & 33.3 & 15.4 \\
\hline Female (32) & $18.63(10.76)$ & $2-44$ & 43.8 & 18.8 & 37.5 \\
\hline Total (71) & $16.27(9.45)$ & $0-44$ & 47.9 & 26.8 & 25.4 \\
\hline Expected $\star$ & $20.18(11.29)$ & & 33.3 & 33.3 & 33.3 \\
\hline \multicolumn{6}{|l|}{ MBI-DP: } \\
\hline Male (39) & $5.21(5.70)$ & $0-19$ & 51.3 & 25.6 & 23.1 \\
\hline Female (32) & $6.72(5.35)$ & $0-17$ & 34.4 & 28.1 & 37.5 \\
\hline Total (71) & $5.89(5.56)$ & $0-19$ & 43.7 & 26.8 & 29.6 \\
\hline Expected $\star$ & $7.03(5.90)$ & & 33.3 & 33.3 & 33.3 \\
\hline \multicolumn{6}{|l|}{ MBI-PA: } \\
\hline Male (39) & $35.31(7.95)$ & $15-48$ & 53.8 & 23.1 & 23.1 \\
\hline Female (32) & $35.44(6.90)$ & $12-48$ & 53.1 & 31.3 & 15.6 \\
\hline Total (71) & $35.37(7.45)$ & $12-48$ & 53.5 & 26.8 & 19.7 \\
\hline Expected $^{\star}$ & $32.52(8.66)$ & & 33.3 & 33.3 & 33.3 \\
\hline
\end{tabular}

${ }^{\star}$ Mean burnout scores and tertile distribution obtained on 748 Italian health workers. ${ }^{30}$

tion of immune cells in the univariate analysis. Also in this case, introduction of all variables did not change the results. Analyses of covariance (ANCOVAs) were finally used by introducing the same covariates to identify differences in immune variables between subjects divided into three different groups of burnout. The burnout scores were stratified into low, medium, and high categories according to the cut off points (tertile distribution) given for Italian health workers. ${ }^{30}$ If an overall analysis of covariance test showed a significant effect, then Tukey's multiple comparison test was performed to determine which categories were different.

\section{Results}

The demographic and professional characteristics of physicians included in the study are shown in table 1 . Subjects ranged in age from 24 to 50, and were almost equally distributed for sex and marital status. Job positions were, 36 regular staff members (assistant or vice head); some of them were also involved in teaching activities, and the rest were in postgraduate training in either anaesthesiology or intensive care, or hygiene and preventive medicine.

Twenty six out of $71(36.6 \%)$ were smokers with a mean (SD) consumption of 13.3 (5.4) cigarettes a day. Most (83.3\%) drank coffee regularly (mean $2.4(1.1)$ cups a day); $73.2 \%$ drank alcohol (73.2\%), whereas only nine $(12.7 \%)$ took drugs regularly. The mean (SD) sleeping time a night was $6.9(0.9)$ hours.
Table 2 presents results of the Maslach burnout inventory scales for the sample as a whole and the sexes separately. No significant differences between men and women were found, although women had higher means on the emotional exhaustion and depersonalisation scales. Compared with the normative data from Italian health workers, ${ }^{30}$ our sample shows a slightly higher score on the personal accomplishment scale and lower on the other scales. This resulted in a higher percentage of subjects with low burnout: emotional exhaustion $=47.9 \%$, depersonalisation $=43.7 \%$, personal accomplishment $=53.5 \%$ (expected $33.3 \%$ ). The trait anxiety score was 37.23 (9.02) (range 20-72), slightly higher in women than men (39.31 (7.11) v 35.51 (10.10), NS), but similar to the normative mean value (41.27 (9.68) v 36.47 (9.60)). ${ }^{31}$

Emotional exhaustion was positively related to depersonalisation and trait anxiety $(r=0.48$ and 0.56 , respectively, $\mathrm{p}<0.001$ ) and negatively to personal accomplishment $(r=-0.42$, $\mathrm{p}<0.001)$. Anxiety was positively associated with depersonalisation $(r=0.26, \mathrm{p}<0.05)$ and negatively with personal accomplishment $(r=-0.37, \mathrm{p}<0.005)$. Lastly, depersonalisation and personal accomplishment were poorly related $(r=-0.24, \mathrm{p}<0.05)$

Stepwise regressions for the burnout scales are presented in table 3 . In the model 1 , the main predictors of emotional exhaustion were job position, marital status, and sex, indicating that high graded, single, and female physicians have higher levels of emotional exhaustion. These results changed in the model 2 , and trait anxiety became the best predictor (positive), followed by the type of work (physicians with patient contact $>$ physicians without contact). The whole model explained $34.1 \%$ of the variation in emotional exhaustion. As expected, the main predictor of depersonalisation was to work with patients, but again a positive influence of anxiety was found. Anxiety was the only variable inversely related to personal accomplishment. By introducing anxiety scores as the dependent variable, only the duration of service entered in the model was positively associated with this personality trait $(F=6.91$, $\mathrm{p}<0.002, R^{2}=0.145$, not shown).

The personal accomplishment scores were positively related to number $(\log )$ and percentage of total lymphocytes $(r=0.273, \mathrm{p}<0.05$;

Table 3 Regression analysis of the factors influencing the scales of the Maslach burnout inventory (MBI)

\begin{tabular}{|c|c|c|c|c|c|c|}
\hline \multirow[b]{3}{*}{ Independent variables } & \multicolumn{6}{|c|}{ Dependent variables } \\
\hline & \multicolumn{2}{|l|}{$M B I-E E$} & \multicolumn{2}{|l|}{$M B I-D P$} & \multicolumn{2}{|l|}{$M B I-P A$} \\
\hline & Model $1 \beta$ & Model $2 \beta$ & Model $1 \beta$ & Model $2 \beta$ & Model $1 \beta$ & Model $2 \beta$ \\
\hline $\operatorname{Sex}(M / F)$ & $+0.323^{\star \star}$ & +0.099 & +0.115 & +0.059 & -0.046 & +0.090 \\
\hline Age (y) & -0.111 & +0.031 & -0.029 & -0.100 & +0.031 & -0.043 \\
\hline Marital status (single, married) & $-0.386^{\star \star}$ & -0.057 & -0.005 & +0.010 & +0.009 & -0.079 \\
\hline Children at home (n) & +0.142 & +0.082 & +0.046 & +0.037 & +0.111 & -0.011 \\
\hline Patient contact (no, yes) & +0.036 & $+0.216^{\star}$ & $+0.339 \star \star$ & $+0.346^{\star \star}$ & +0.098 & -0.017 \\
\hline Job position ( 5 categories $\dagger$ ) & $-0.574^{\star \star \star}$ & -0.147 & -0.128 & -0.047 & +0.351 & +0.113 \\
\hline Duration of service $(y)$ & -0.069 & +0.083 & +0.082 & -0.004 & +0.037 & -0.038 \\
\hline STAI-Y2 (scores) & - & $+0.565^{\star \star \star}$ & - & $+0.274^{\star}$ & - & $-0.368^{\star \star}$ \\
\hline$F$ ( $p$ value) & $9.11(<0.001)$ & $19.11(<0.001)$ & $8.94(<0.005)$ & $7.97(<0.002)$ & $0.54(\mathrm{~ns})$ & $10.79(<0.002)$ \\
\hline Adjusted $\mathrm{R}^{2}$ & 0.258 & 0.341 & 0.102 & 0.166 & 0.048 & 0.123 \\
\hline
\end{tabular}

${ }^{\star} \mathrm{p}<0.05 ;{ }^{\star \star} \mathrm{p}<0.005 ;{ }^{\star \star \star} \mathrm{p}<0.001$.

†Vice head, assistant, 4 y, 3 y, 2 y postgraduate. 

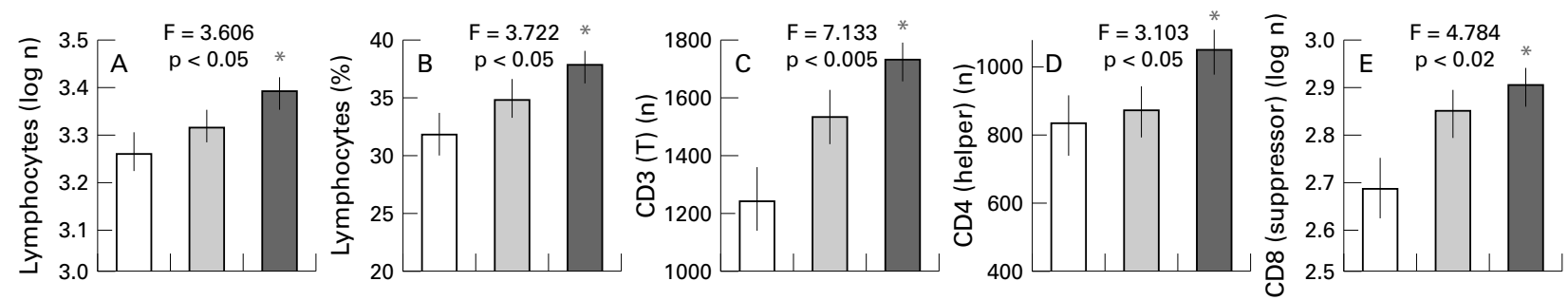

Personal accomplishments:

$\square$ Low; $\square$ Medium; $\square$ High

Immunological variables showing significant modifications with personal accomplishment. Mean (SE) corrected for sex, duration of service, job position, and smoking habits (ANCOVA). Low, medium, and high levels of personal accomplishment are defined according to the cut off points (tertile distribution) obtained for Italian health workers. ${ }^{30}$

$r=0.385, \mathrm{p}<0.001$; respectively), number of CD3 $(r=0.319, \quad \mathrm{p}<0.01), \mathrm{CD} 4 \quad(r=0.312$, $\mathrm{p}<0.01)$, and $(\log ) \operatorname{CD} 8(r=0.256, \mathrm{p}<0.05)$.

Mean corrected values (ANCOVA) of the immune variables in the subjects grouped according to the personal accomplishment categories (low, medium, high) are presented in the figure. This shows a significant positive relation between personal accomplishment and each immune variable. At the final analysis, physicians with high personal accomplishment (score $\leqslant 29$ ) had a significantly higher number and percentage of lymphocytes, number of $\mathrm{T}$ cells (CD3), T helper cells (CD4), and T suppressor cells (CD8) compared with those with low personal accomplishment (score $\geqslant 37$ ). As stepwise multiple regression analyses confirmed these relations after controlling for the same potential confounders, only data from ANCOVA have been presented.

No other immune variable was significantly related to personal accomplishment or to the other Maslach burnout inventory scales.

\section{Discussion}

In our group of physicians, professional characteristics, sociodemographic variables, and trait anxiety were significant predictors of burnout. A large portion of the variation of the emotional exhaustion scale was associated with marital status, sex, and job characteristics (qualification, working activity), whereas only the type of work explained a portion of variation in depersonalisation. The risk profile obtained suggests that to have a position of high responsibility, to be single, and to be female are risk factors for higher levels of emotional exhaustion, whereas depersonalisation is strictly related to a repetitive contact with patients. A similar risk profile was drawn up in a random sample of professionals in Spain: to be female, $>44$ years old, with no stable partner, with $>29$ years of service, working in a specialised department, with a high number of patients under her responsibility, and with a working week of $36-40$ hours. $^{35}$ As in other studies, age and years of practice were not significant predictors of burnout, ${ }^{79}$ whereas an association between emotional exhaustion and certain personal characteristics such as age and marital status have been already reported. ${ }^{36}$

This study highlights the relation between burnout and trait anxiety, an enduring personal quality. Indeed, anxiety contributed signifi- cantly to the scores for emotional exhaustion, depersonalisation (positively), and personal accomplishment (negatively), even after the other demographic and professional variables were controlled. Significant associations between anxiety and the Maslach burnout inventory scales, particularly emotional exhaustion, have been already described in a longitudinal study which underlines the contribution of personality to the experience of burnout. ${ }^{28}$ Significant associations between burnout, personal accomplishment, anxiety, and depression were also found in HIV and AIDS healthcare workers, in psychiatric hospital staff, and other health workers. ${ }^{29} 3738$ These findings suggest that enduring personal characteristics, and not only situational variables, should be adequately considered in the prevention, intervention, and treatment of burnout.

The average level of burnout in the sample examined was slightly lower than that expected for the Italian healthcare personnel. Explanations include the relatively young age of the group examined and the selection of physicians with different levels of stress in terms of contact with patients. Both factors have been carefully matched. The young age was selected to avoid possible changes in both immune variables and burnout levels, ${ }^{15}{ }^{36}$ and to have a more homogeneous group for lifestyle and family, education, and attitude towards work. The inclusion of physicians with different qualification and type of work have been considered of particular value in obtaining a larger range of burnout scores.

For the relation between burnout and immune variables, in our group of relatively young physicians there is evidence that high personal accomplishment scorers are more likely to have lymphocyte and $\mathrm{T}$ cell counts above the normal range. The meaning of this is not clear, although we could speculate that the feeling of reward and success to receive pleasure and gratification from work may help to stimulate the immune defences of healthcare workers. By contrast, there is no evidence that to work hard, to feel tired from work, and to have a cynical reaction towards patient care is actually associated with immunosuppression. As it has been suggested that chronic psychological stress negatively modifies immunological responses, ${ }^{16}{ }^{23-25}$ these findings support the view that burnout, at least in our group, represents a defensive reaction to high clinical 
workloads. Undoubtedly, in our physicians high emotional exhaustion and depersonalisation scores seem to be counterbalanced by high personal accomplishment (table 2), and it is well known that hard work may lead to burnout but achievement through work that is held in high regard by others, and doing work that is highly valued correlate negatively with job stress. ${ }^{12} 3639$

Personal accomplishment has been positively associated with various personality dimensions - such as extraversion, agreeableness, and conscientiousness - and negatively with vulnerability ${ }^{28}{ }^{40}$ and anxiety, as in our sample. However, in our study personal accomplishment was not related to demographic and professional characteristics, nor with job satisfaction evaluated by others. ${ }^{14}$ Based on these findings, it seems reasonable to conclude that personal accomplishment has little to do with work environment and is more closely associated with those personality dimensions that underlie wellbeing. A positive influence of individual psychological characteristics on the immune system has been previously reported: subjects who were emotionally stable and not anxious responded to a natural psychological stressor (academic examination) with increased natural killer cell activity, whereas the opposite was found in subjects with low emotional stability and high anxiety. ${ }^{26}$

Our results indicate that, independently of the workload, to evaluate oneself positively, to be hard working, active, agreeable, and motivated, particularly with regard to work in the health services, is associated with selective and positive effects on immune cell counts in peripheral blood. Additional research is needed to specify the mechanism involved and to investigate the health effects associated with these findings. Actually, this question cannot be answered by the available data and definite conclusions cannot be drawn.

This work was supported by a grant from the Italian MURST (Ministero dell'Università e della Ricerca Scientifica e Tecnologica), $60 \%$ funds. The authors thank Dr Raffaele Stacca, Dr Gian Carlo Barbieri, Dr Carlo Montanari, heads of anaesthesiology and intensive care for their cooperation, and colleagues for their assistance in making this project possible.

1 Harrington JM, Calvert IA. Research priorities in occupational medicine: a survey of United Kingdom personnel managers. Occup Environ Med 1996;53:642-4.

2 Houtman ILD, Goudswaard A, Dhondt S, et al. Dutch monitor on stress and physical load: risk factors, consequences, and preventive action. Occup Environ Med quences, and $1998 ; 55: 73-83$.

3 Woodward CA, Shannon HS, Cunningham C, et al. The impact of re-engineering and other cost reduction strategies on the staff of a large teaching hospital. A longitudinal study. Med Care 1999;37:556-69.

4 Maslach C, Jackson SE. Maslach burnout inventory manual, 2nd ed. Palo Alto: Consulting Psychologists Press, 1986.

5 Deckard G, Meterko M, Field D. Physician burnout: an examination of personal, professional, and organizational relationships. Med Care 1994;32:745-54.

6 Fields AI, Cuerdon TT, Brasseux CO, et al. Physician burnout in paediatric critical care medicine. Crit Care Med 1995;23:1425-9.

7 Kirwan M, Armstrong D. Investigation of burnout in a sample of British general practitioners. Br F Gen Pract 1995;45: 259-60.

8 Catalan J, Burgess A, Pergami A, et al. The psychological impact on staff of caring for people with serious diseases: the case of HIV infection and oncology. 7 Psychosom Res 1996;40:425-35.

9 Goldberg R, Boss RW, Chan L, et al. Burnout and its correlates in emergency physicians: four years' experience with a wellness booth. Acad Emerg Med 1996;3:1156-64.

10 Guntpalli KK, Fromm RE Jr. Burnout in the internistintensivist. Intensive Care Med 1996;22:625-30.

11 Prosser D, Johnson S, Kuipers E, et al. Mental health, burnout, and job satisfaction among hospital and communitybased mental health staff. Br F Psychiatry 1996;169:334-7.

12 Visintini R, Campanini E, Fossati A, et al. Psychological stress in nurses' relationships with HIV-infected patients: the risk of burnout syndrome. AIDS Care 1996;8:183-94

13 Watson P, Feld A. Factors in stress and burnout among paediatric nurses in a general hospital. Nursing Praxis in New Zealand 1996;11:38-46.

14 Humphris G, Lilley J, Kaney S, et al. Burnout and stress-related factors among junior staff of three dental hospital specialties. Br Dent f 1997;183:15-21.

15 Iacovides A, Fountoulakis K, Moysidou C, et al. Burnout in nursing staff: a clinical syndrome rather than a psychological reaction? Gen Hosp Psychiatry 1997;19:419-28.

16 Nakamura H, Nagase H, Yoshida $M$, et al. Natural killer (NK) cell activity and NK cell subsets in workers with a tendency of burnout. $\mathcal{F}$ Psychosom Res 1999;46:569-78.

17 O'Leary A. Stress, emotion, and human immune function. Psychol Bull 1990;108:363-82.

18 Ader R, Cohen N. Psychoneuroimmunology: conditioning and stress. Ann Rev Psychol 1993;44:53-85.

19 Herbert TB, Cohen S. Stress and immunity in humans: a meta-analytic review. Psychol Med 1993;55:364-79.

20 Schedlowski M, Jacobs R, Alker J, et al. Psychophysiological, neuroendocrine and cellular immune reactions under psychological stress. Neuropsychobiology 1993;28:87-90.

21 Ader R, Cohen N, Felten D. Psychoneuroimmunology: interactions between the nervous system and the immune system. Lancet 1995;345:99-103.

22 Kemeny ME, Cohen F, Zegans LS, et al. Psychological and immunological predictors of genital herpes recurrence. Psychosom Med 1989;51:195-208.

23 Christensen AJ, Edwards DL, Wiebe JS, et al. Effect of verbal self-disclosure on natural killer cell activity: moderating influence of cynical hostility. Psychosom Med 1996;58:1505.

24 Koh KB, Lee BK. Reduced lymphocyte proliferation and interleukin-2 production in anxiety disorders. Psychosom Med 1998;60:479-83.

25 Scanlan JM, Vitaliano PP, Ochs H, et al. CD4 and CD8 counts are associated with interactions of gender and psychosocial stress. Psychosom Med 1998;60:644-53.

26 Borella P, Bargellini A, Rovesti S, et al. Emotional stability, anxiety, and natural killer activity under examination stress. Psychonuroendocrinology 1999;24:613-27.

27 Jung W, Irwin M. Reduction of natural killer cytotoxic activity in major depression: interaction between depression and cigarette smoking. Psychosom Med 1999;61:263-70.

28 Piedmont RL. A longitudinal analysis of burnout in the health care setting: the role of personal dispositions. $\mathcal{F}$ Pers Assess 1993;61:457-73.

29 Bellani ML, Furlani F, Gnecchi M, et al. Burnout and related factors among HIV/AIDS health care workers. AIDS Care 1996;8:207-21.

30 Maslach C, Jackson S. Maslach burnout inventory. Manuale. (S Sirigatti, C Stefanile. Italian version.) Firenze: OS Organizzazioni Speciali, 1993.

31 Spielberger CD. Inventario per l'Ansia di Stato e di Tratto, forma Y. Manuale. (L Pedrabissi, M Santinello. Italian version.) Firenze: OS Organizzazioni Speciali, 1989.

32 Pagliaro P, Del Vecchio L, Girotto M, et al. Lymphocyte subsets in Italian blood donors. La Trasfusione del Sangue 1996;41:1-9.

33 Borella P, Bargellini A, Salvioli S, et al. The use of non-radioactive chromium as an alternative to ${ }^{51} \mathrm{Cr}$ in NK assay. F Immunol Methods 1995;186:101-10.

34 Borella P, Bargellini A, Salvioli S, et al. Use of flameless atomic absorption spectroscopy in immune cytolysis for non-radioactive determination of killer cell activity. Clin Chem 1996;42:319-25.

35 Atance-Martinez JC. Epidemiologic aspects of the burnout syndrome in hospital personnel. Revista Espanola de Salud Publica 1997;71:293-303.

36 Ramirez AJ, Graham J, Richards MA, et al. Mental health of hospital consultants: the effects of stress and satisfaction at work. Lancet 1996;347:724-8.

37 Corrigan PW, Holmes EP, Luchins D. Burnout and collegial support in state psychiatric hospital staff. $\mathcal{F}$ Clinical Psychology 1995;51:703-10

38 Turnipseed DL. Anxiety and burnout in the health care work environment. Psychol Rep 1998;82:627-42.

39 Knoop R. Relieving stress though value-rich work. $\mathcal{f}$ Soc Psychol 1994;134:829-36.

40 Deary IJ, Blenkin H, Agius RM, et al. Models of job-related stress and personal achievement among consultant doctors. Br f Psychol 1996;87:3-29. 